\title{
Testes sensoriais de aceitação da beterraba vermelha (Beta vulgaris ssp. vulgaris L.), cv. Early Wonder, minimamente processada e irradiada \\ Sensory acceptance tests of red beet (Beta vulgaris ssp. vulgaris L.), cv. Early Wonder, minimally processed and irradiated
}

\author{
Nilber Kenup HERNANDES ${ }^{1 *}$, Regina Celi Cavestré CONEGLIAN ${ }^{2}$, Ronoel Luiz de Oliveira GODOY ${ }^{3}$, \\ Helio de Carvalho VITAL ${ }^{1}$, Murillo FREIRE JUNIOR ${ }^{3}$
}

\begin{abstract}
Resumo
A beterraba vermelha (variedade hortícola, cultivar Early Wonder) foi cultivada na área experimental do Departamento de Fitotecnia do Instituto de Agronomia da Universidade Federal Rural do Rio de Janeiro. Após a colheita, as raízes foram minimamente processadas, embaladas e submetidas a diferentes doses de radiação gama $(0 ; 0,5 ; 1,0$; e $1,5 \mathrm{kGy})$, sendo em seguida armazenadas, por 20 dias, a $8{ }^{\circ} \mathrm{C}$. Durante 0 período de armazenamento, aos: 1, 9, 13 e 20 dias, foram realizados testes sensoriais, nos quais 12 avaliadores julgaram a aparência e o aroma segundo uma escala hedônica. Os resultados indicaram que as amostras irradiadas com doses de 1,0 e 1,5 kGy mantiveram-se dentro dos padrões de aceitabilidade por 20 dias. Alem disso, foi também observado que a avaliação do aroma permitiu uma melhor discriminação, dos efeitos das diferentes doses de radiação sobre a aceitação do produto.

Palavras-chave: processamento mínimo; irradiação de alimentos; extensão da vida útil; qualidade sensorial; aroma.
\end{abstract}

\begin{abstract}
Red beet (Early Wonder) was cultivated in an experimental area of the Department of Fitotecnia at the Institute of Agronomy at the Rural Federal University of Rio de Janeiro (UFRRJ). After harvest, the roots were minimally processed; packed; exposed to different doses of gamma radiation $(0 ; 0.5 ; 1.0$ and $1.5 \mathrm{kGy})$ and stored for 20 days at $8.0^{\circ} \mathrm{C}$. Sensory evaluations were performed $1 ; 9 ; 13$ and 20 days after irradiation by 12 testers who rated the overall appearance and aroma on a hedonic scale. The results indicated that the irradiated samples remained within the limit of acceptance for 20 days. In addition, the aroma was found to be a more sensitive indicator of the effect of different doses of radiation to the acceptance of the product.

Keywords: minimum processing; food irradiation; shelf-life extension; sensory quality; aroma.
\end{abstract}

\section{Introdução}

A demanda por produtos prontos para o consumo, como os produtos minimamente processados, tem crescido em todo o mundo desde seu surgimento, há mais de 30 anos, nos Estudos Unidos da América. No Brasil, embora o mercado ainda seja considerado incipiente ${ }^{9}$, já se observa um crescimento considerável na oferta destes produtos, voltada principalmente para o mercado das cozinhas industriais e para os grandes centros consumidores. Essa tendência é conseqüência da crescente conscientização dos consumidores que valorizam, cada vez mais, produtos que, além de oferecerem maior comodidade e praticidade na hora do preparo ou consumo, conservem seu sabor natural e seu valor nutritivo.

O processamento mínimo de vegetais tem como objetivo oferecer um produto pronto para o consumo que conserve o frescor, garanta a manutenção das qualidades sensoriais e seja seguro, isto é, não apresente riscos à saúde dos consumidores. Para melhor atender a essas exigências, vários estudos têm sido realizados ${ }^{4,14,16,20,24-26,28}$, sendo que alguns deles têm proposto a irradiação como opção para promover a extensão da vida útil de produtos minimamente processados $3,10,17,19$.

Seção de Defesa Nuclear do Centro Tecnológico do Exército. Av. das Américas,

28705, Guaratiba, CEP 23020-040, Rio de Janeiro - RJ, Brasil,

E-mail:nilberkenup@ctex.eb.br

Departamento de Fitotecnia, Instituto de Agronomia,

Universidade Federal Rural do Rio de Janeiro - UFRRJ,

Rio de Janeiro - RJ, Brasil

3 Embrapa Agroindústria de Alimentos, Brasil

* A quem a correspondência deve ser enviada
A eficácia e a segurança do processo de irradiação, objetivando a extensão da vida útil de vários tipos de alimentos, já foram extensivamente comprovadas ${ }^{8,11,12,18,21,22}$. Esse tratamento é indicado quando se deseja diminuir, inativar ou mesmo eliminar a carga microbiológica residual que permanece no alimento após os processos de sanitização, bem como inibir processos fisiológicos enzimáticos de deterioração do produto. Sua utilização, contudo, deve ser bem avaliada, principalmente no que se refere às qualidades sensoriais do produto, uma vez que a irradiação pode prejudicar algumas características sensoriais do alimento. Por outro lado, sabe-se que esses efeitos podem ser correlacionados com as doses de radiação aplicadas e que cada alimento comporta-se de modo distinto ${ }^{8,12}$. Por conseguinte, antes de se adotar comercialmente o tratamento como meio de extensão da vida útil de um produto, torna-se necessária a realização de estudos que avaliem os seus possíveis efeitos sobre a aceitação do produto irradiado.

Nas análises sensórias, são utilizados os órgãos dos sentidos humanos para realização de medidas multidimensionais integradas que objetivam determinar a aceitação de um produto por parte do consumidor ${ }^{2}$. A aceitação ou rejeição de um alimento depende de suas propriedades sensoriais, sendo assim, uma escala hedônica pode ser utilizada como método de avaliação da aceitação do consumidor ${ }^{5}$. Nesse tipo de avaliação, podem ser medidos, tanto fatores intrínsecos (como a aparência e o aroma, investigados neste trabalho), como fatores extrínsecos (como embalagem, informações no rótulo, etc.). 
Ao se avaliar a aparência, na verdade, investiga-se um amplo conjunto de características distintas, como: a cor (fator de maior relevância na aparência); dimensões do produto; tipos de corte; etc. Já em relação ao aroma, o que é avaliado é o conjunto de compostos aromáticos voláteis que são naturalmente liberados pelo produto. Os processos de deterioração, tanto os enzimáticos como os provocados por microorganismos, acabam por liberar compostos aromáticos característicos que são facilmente detectáveis pelo olfato. Vários trabalhos dedicados a alimentos de origem vegetal, minimamente processados, têm se utilizado das análises sensoriais como ferramenta para determinar a aceitação desses produtos ${ }^{4-7}$. Em um dos trabalhos mais recentes nessa área, KLUGE et al. ${ }^{13}$ usaram a análise sensorial para determinar a conservação de beterrabas minimamente processadas, baseando-se em sua qualidade visual. Esses autores concluíram que: "A beterraba minimamente processada em fatias ou cubos apresenta conservação de no máximo 4 dias a $5^{\circ} \mathrm{C}$, enquanto a minimamente processada em retalhos apresenta 6 dias de conservação”.

A beterraba, planta da família Chenopodiaceae, pertence à espécie Beta vulgaris L. e, segundo LANGE et al., ${ }^{15}$ se divide em três subespécies:

a) Beta vulgaris ssp. adanesis, grupo distinto de plantas semi-anuais, com características morfológicas específicas, que apresentam um grande declínio na autofertilização;

b) Beta vulgaris ssp. maritima, formada por um grande complexo de tipos morfológicos que ocorrem em uma vasta área geográfica, e cujas diferenças são insuficientes para estabelecer outras subdivisões; e

c) Beta vulgaris ssp. vulgaris, que agrupa todas as cultivares já domesticadas. Ainda segundo LANGE et al., ${ }^{15}$ todas as cultivares da subespécie Beta vulgaris ssp. vulgaris (conhecidas até então) podem ser subdivididas em quatro grupos: beterrabas folhosas (Leaf Beet Group), cultivares nas quais as partes comestiveis são as folhas e os pecíolos, pois suas raízes não exibem diâmetro significativamente aumentado; beterrabas açucareiras (Sugar Beet Group), em sua maioria de coloração branca e que são cultivadas nos Estados Unidos da América e no continente europeu para produção de açúcar; beterrabas forrageiras (Fodder Beet Group), cultivares destinadas à alimentação dos rebanhos das regiões já citadas; e beterrabas hortícolas (Garden Beet Group), único grupo cultivado comercialmente no Brasil formado por cultivares que apresentam a uma parte tuberosa comestível.

A beterraba vermelha (hortícola), além de ser muito nutritiva, possui forte apelo sensorial, devido à sua cor vermelhointensa. Esse aspecto sensorial pode ser uma das razões que explicam o crescimento, no mercado brasileiro, do consumo de beterraba minimamente processada, que está sendo impulsionado pelo segmento de saladas prontas (fresh-cut), cada vez mais presentes nas gôndolas dos supermercados, principalmente nos grandes centros. Sua coloração é decorrente da presença das betacianinas (pigmentos vermelhos que, juntamente com as betaxantinas, de coloração amarela, pertencem ao grupo das betalaínas). Esses pigmentos possuem atividade antioxidante ${ }^{1}$ e são hidrossolúveis. Isso acarreta a descoloração da beterraba minimamente processada, o que se constitui em um problema tecnológico a ser contornado, pois prejudica a aceitação e diminui a vida útil do produto ${ }^{13,28}$. Visando à solução desse problema, preconiza-se adicionar ácido cítrico (2000 $\left.\mu \mathrm{g} . \mathrm{L}^{-1}\right)$ à água de sanitização durante o processamento ${ }^{23,29}$.

O presente trabalho tem como objetivo avaliar a aceitação da beterraba vermelha minimamente processada e irradiada, por meio de análises sensoriais da aparência e aroma, num período de 20 dias de armazenamento a $8{ }^{\circ} \mathrm{C}$.

\section{Material e métodos}

As beterrabas utilizadas neste estudo foram cultivadas no segundo semestre de 2005, no campo experimental do Departamento de Fitotecnia do Instituto de Agronomia da Universidade Federal Rural do Rio de Janeiro (latitude $22^{\circ} 45^{\prime}$ 50,6” Sul, longitude $43^{\circ} 41^{\prime}$ 53,9” Oeste), Seropédica, RJ. A cultivar usada foi a Early Wonder. Essa cultivar, originária dos Estados Unidos da América, é atualmente a mais plantada no Brasil e suas principais características são raiz com formato globular e forte coloração vermelha. É também, uma variedade considerada precoce, com início da colheita variando entre 60 e 70 dias após o transplante das mudas e, além disso, apresenta uma excelente adaptação às diversas regiões de cultivo.

As beterrabas foram colhidas aos 73 dias e transferidas para a Planta Piloto de Fisiologia e Tecnologia Pós-colheita da Embrapa Agroindústria de Alimentos em Guaratiba, Município do Rio de Janeiro, sendo mantidas em câmara fria $\left(5{ }^{\circ} \mathrm{C}\right)$ até o dia seguinte, quando foram processadas. No processamento, foi seguido o fluxograma mostrado na Figura 1. Após o processamento mínimo, as amostras foram divididas em quatro grupos, a saber: 0 (controle, não irradiado) e amostras a serem irradiadas com doses de 0,5; 1,0; e 1,5 kGy. Todas as amostras foram mantidas em câmara fria $\left(5^{\circ} \mathrm{C}\right)$ até o dia seguinte, quando foram transferidas para as instalações da Seção de Defesa Nuclear do Centro Tecnológico do Exército (também localizado em Guaratiba, Município do Rio de Janeiro, RJ), para serem irradiadas, utilizando-se para esse fim o Irradiador de Pesquisa do CTEx, que atualmente gera uma taxa de dose máxima de $1,8 \mathrm{kGy} \cdot \mathrm{h}^{-1}$ de radiação gama (energia de 0,662 MeV), emitida por sua fonte de ${ }^{137} \mathrm{Cs}$ (com atividade da ordem de $46 \mathrm{kCi}^{27}$ ). Depois da irradiação, as amostras foram mantidas em câmaras de refrigeração do tipo BOD a uma temperatura de $8 \pm 0,5^{\circ} \mathrm{C}$, sendo avaliadas por um período de 20 dias.

As análises sensoriais foram realizadas por avaliadores voluntários não treinados. Cada avaliador recebeu um formulário para julgamento dos atributos aparência e aroma (Figura 2) e uma bandeja contendo quatro amostras codificadas (com aproximadamente 25 g cada), sendo uma amostra para cada dose $(0 ; 0,5 ; 1,0$; e 1,5 kGy). As amostras foram avaliadas em quatro ocasiões: 1, 9, 13 e 20 dias após a irradiação, sendo que, em cada uma delas, participaram doze avaliadores.

$\mathrm{Na}$ análise dos resultados das avaliações, foram calculadas as médias de cada atributo. Isso foi possível atribuindo-se à escala hedônica notas que variaram de 1 até 9 . Ao termo "Desgostei extremamente" foi atribuído o valor 1 e ao termo "Gostei 


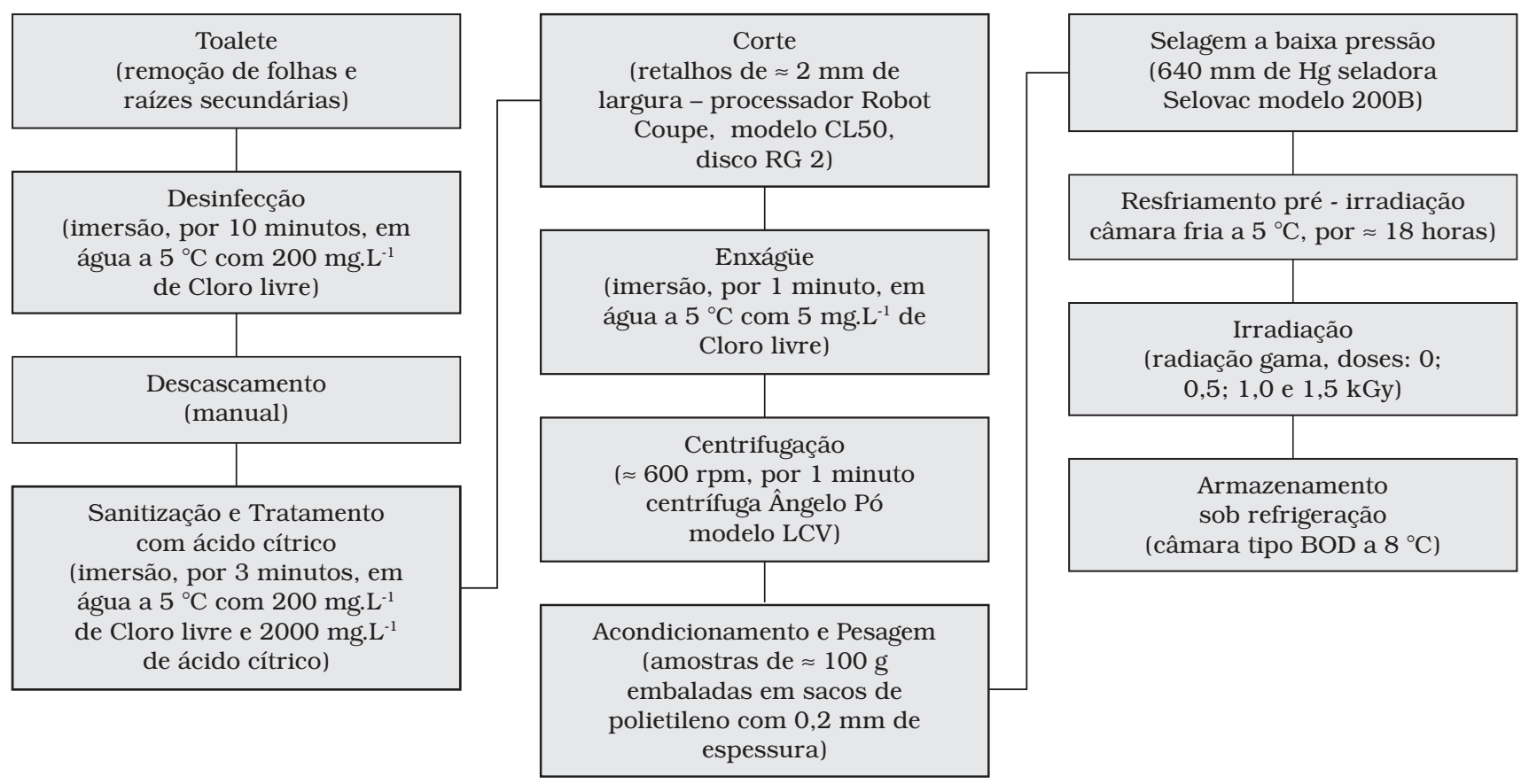

Figura 1. Fluxograma das operações unitárias empregadas na elaboração de beterrabas minimamente processadas e irradiadas.

\begin{tabular}{|c|c|c|c|c|c|c|c|c|}
\hline \multicolumn{9}{|c|}{ Análise Sensorial de beterraba minimamente processada } \\
\hline \multicolumn{6}{|l|}{ Nome: } & \multicolumn{3}{|c|}{ Data: } \\
\hline \multicolumn{9}{|c|}{$\begin{array}{l}\text { Você gosta de beterraba? ( ) sim ( ) não } \\
\text { Você costuma consumir beterraba crua com fr }\end{array}$} \\
\hline \multicolumn{9}{|c|}{$\begin{array}{l}\text { Por favor, avalie cada uma das amostras, levando em consideração os seguintes } \\
\text { atributos: Aparência (Apa) e Aroma (Aro). Utilize a escala para descrever o quanto } \\
\text { você gostou ou desgostou do produto. Marque, na coluna correspondente ao } \\
\text { atributo avaliado, a posição da escala que melhor reflete sua opinião. }\end{array}$} \\
\hline \multicolumn{9}{|l|}{ Código da amostra } \\
\hline Atributo avaliado & Apa & Aro & Apa & Aro & Apa & Aro & Apa & Aro \\
\hline \multicolumn{9}{|l|}{ Gostei extremamente } \\
\hline \multicolumn{9}{|l|}{ Gostei muito } \\
\hline \multicolumn{9}{|l|}{ Gostei moderadamente } \\
\hline \multicolumn{9}{|l|}{ Gostei ligeiramente } \\
\hline \multicolumn{9}{|l|}{ Indiferente } \\
\hline \multicolumn{9}{|l|}{ Desgostei ligeiramente } \\
\hline \multicolumn{9}{|l|}{ Desgostei moderadamente } \\
\hline \multicolumn{9}{|l|}{ Desgostei muito } \\
\hline Desgostei extremamente & & & & & & & & \\
\hline
\end{tabular}

Figura 2. Formulário utilizado nas análises sensoriais de beterrabas minimamente processadas e irradiadas.

extremamente" foi atribuído o valor 9. O limite inferior da média para a aceitação do produto foi estabelecido em 5. Os dados foram submetidos à análise de variância e para a comparação entre as médias obtidas foi aplicado o teste Tukey ao nível de $5 \%$ de significância.

\section{Resultados e discussão}

Durante o período de armazenamento, foram observadas diferenças significativas quanto à aceitação das beterrabas minimamente processadas e irradiadas com diferentes doses.

\subsection{Aparência}

Foi observado que, relativamente ao atributo aparência (Figura 3), as amostras irradiadas com 1,0 e 1,5 kGy mantiveram-se aceitáveis até o fim do período de avaliação (20 dias), enquanto que a amostra que recebeu uma dose de 0,5 kGy foi rejeitada na avaliação feita aos 20 dias. O gráfico também permite concluir que a amostra controle $(0 \mathrm{kGy})$ provavelmente teria sido rejeitada em uma avaliação que fosse realizada aos 15 dias. 


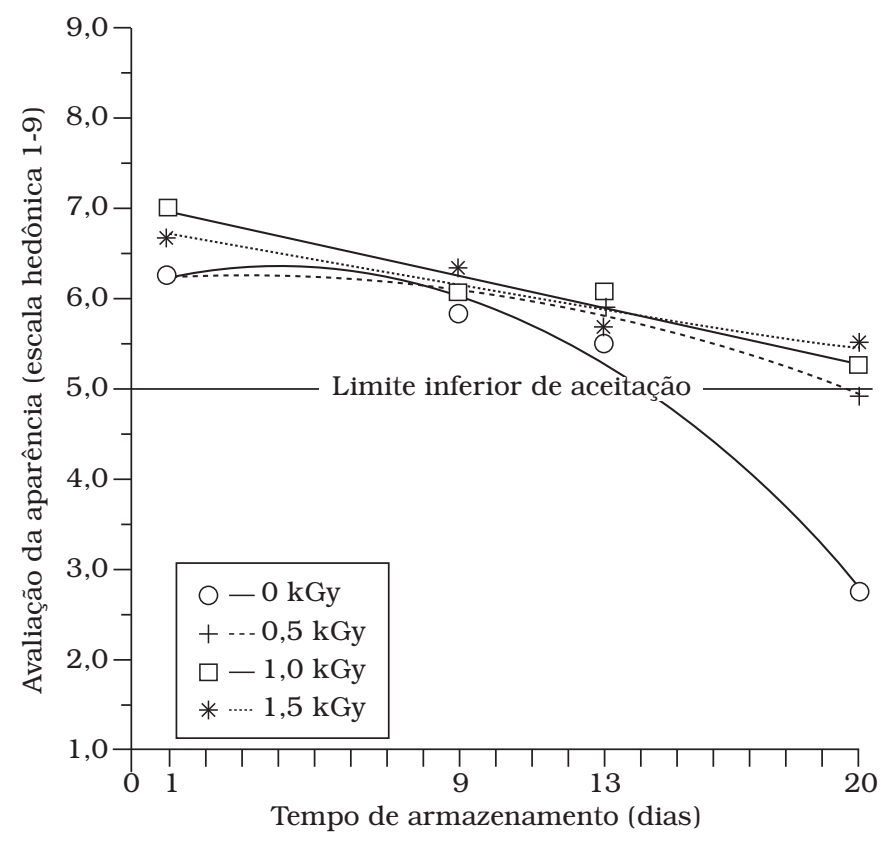

Figura 3. Gráfico da aceitação, com base na avaliação da aparência da beterraba vermelha, minimamente processada e irradiada com diferentes doses durante um período de 20 dias de armazenamento, a $8{ }^{\circ} \mathrm{C}$.

As diferenças entre os resultados relativos aos diferentes tratamentos podem ser atribuídas principalmente às diferentes concentrações da microbiota deteriorante residual (principalmente na amostra controle, $0 \mathrm{kGy}$, e na amostra que recebeu a menor dose, 0,5 kGy), que tende a aumentar ao longo do tempo, comprometendo a aparência dos produtos minimamente processados.

\subsection{Aroma}

Com relação à aceitação das amostras com base no aroma (Figura 4), as mesmas considerações feitas em relação à aparência podem ser tecidas. Cabe ressaltar, entretanto, que este atributo mostrou-se mais sensível ao efeito de diferentes doses de radiação durante o período de armazenamento.

\section{Conclusões}

Com base nos resultados obtidos, pode-se concluir que, quando a beterraba vermelha minimamente processada é irradiada com doses de 1,0 ou 1,5 kGy e armazenada sob refrigeração $\left(8^{\circ} \mathrm{C}\right)$, tanto sua aparência quanto seu aroma se mantêm acima do limite de aceitação por no mínimo 20 dias.

Além disso, foi também observado que a avaliação do aroma mostrou-se mais sensível no que se refere à percepção dos efeitos, da aplicação de diferentes doses de radiação gama, sobre a aceitação do produto.

\section{Agradecimentos}

À Divisão DQBN do CTEx, ao Departamento de Fitotecnia da UFRRJ e à EMBRAPA Agroindústria de Alimentos, pelo apoio decisivo para a realização deste trabalho.

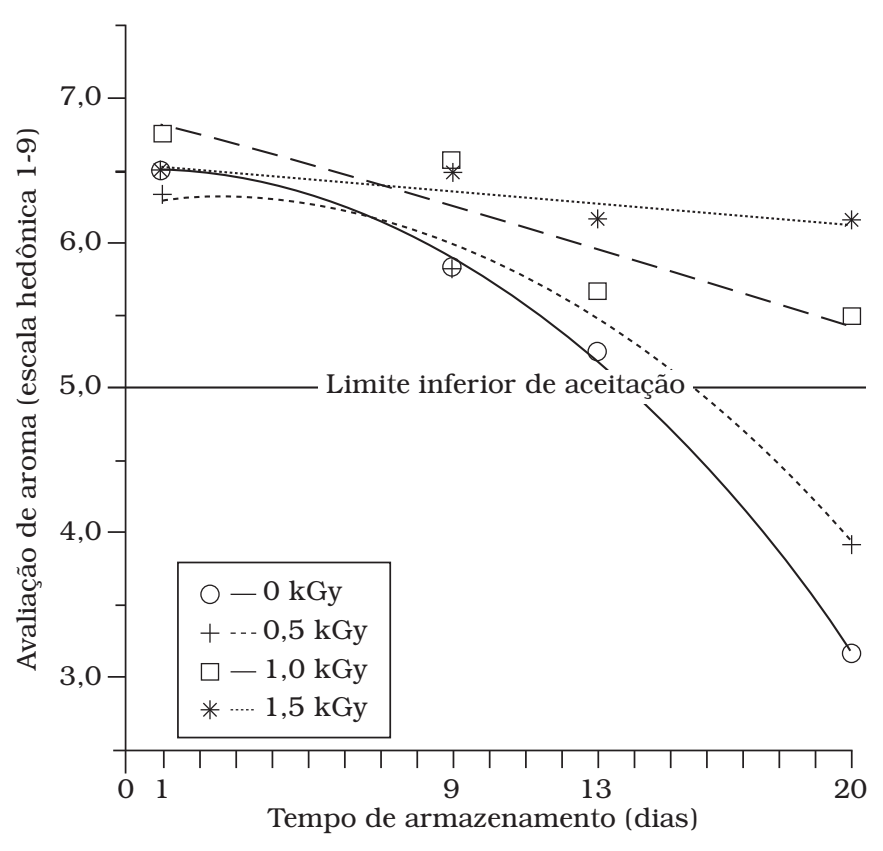

Figura 4. Gráfico da aceitação, com base na avaliação do aroma da beterraba vermelha, minimamente processada e irradiada com diferentes doses durante um período de 20 dias de armazenamento, a $8{ }^{\circ} \mathrm{C}$.

A todos os servidores (civis e militares) da Divisão DQBN do CTEx que participaram como avaliadores nos testes sensoriais.

Às graduandas em Engenharia Agronômica da UFRRJ: Aline Oliveira Ângelo, Bianca Gonçalo Meyrelles e Fernanda Fátima Delgado de Almeida que, com muita dedicação, participaram das etapas do processamento mínimo de beterraba.

\section{Referências bibliográficas}

1. BOBBIO, P. A.; BOBBIO, F. O. Química do Processamento de Alimentos. Livraria Varela, São Paulo, SP, 2001, $3^{\circ}$ edição, 143 p. il. ISBN: 85-85519-12-6.

2. CARDEllo, H. M. A. B.; CARDEllo, L. Teor de vitamina C, atividade de escorbato oxidase e perfil sensorial de manga (Mangífera índica L.) var. haden, durante o amadurecimento. Ciência e Tecnologia de Alimentos, v. 18, n. 2, p. 211-217, abr./jun. 1998.

3. CHAUDRY, M. A.; BIBI, N.; KHAN, M.; KHAN, M.; BADSHAH, A.; QURESHI, M. J. Irradiation treatment of minimally processed carrots for ensuring microbiological safety. Radiation Physics and Chemistry, v. 71, p. 169-173, 2004.

4. DAMASCENO, K. S. F. S. C.; ALVES, M. A.; MENDONÇA, S. C.; GUERRA, N. B.; STAMFORD, T. L. M. Melão minimamente processado: Um controle de qualidade. Ciência e Tecnologia de Alimentos, v. 25, n. 4, p. 651-658, out./dez. 2005.

5. DANTAS, M. I. S.; MINIM, V. P. R.; PUSCHMANN, R.; CARNEIRO, J. D. S.; BARBOSA, R. L. Mapa de preferência de couve minimamente processada. Horticultura Brasileira, v. 22, n. 1, jan./mar. 2004.

6. DANTAS, M. I. S.; DELIZA, R; MINIM, V. P. R.; HEDDERLEY, D. Avaliação da intenção de compra de couve minimamente 
processada. Ciência e Tecnologia de Alimentos, v. 25, n. 4 , p. 762-767, out./dez. 2005.

7. DELlA TORRE, J. C. M.; RODAS, M. A. B.; BADOLATO, G. G.; TADINI, C. C. Perfil sensorial e aceitação de suco de laranja pasteurizado minimamente processado. Ciência e Tecnologia de Alimentos, v. 23, n. 2, p. 105-111, mai. /ago. 2003.

8. DIEHL, J. F. - Safety of Irradiated Foods - Marcel Dekker Inc, New York, 1990, ISBN: 0-8247-8137-6. 345 p, 22 cm.

9. EMBRAPA - Empresa Brasileira de Pesquisa Agropecuária - Embrapa Hortaliças; SEBRAE - Serviço Brasileiro de Apoio às Micro e Pequenas Empresas - Iniciando um Pequeno Grande Negócio Agroindustrial: Hortaliças Minimamente Processadas - Série Agronegócios, Embrapa Informações Tecnológicas, Brasília, DF, 2003. 133 p. il.

10. GOULARTE, L.; MARTINS, C. G.; MORALES-AIZPURÚA, I. C.; DESTRO, M. T.; FRANCO, B. D. G. M.; VIZEU, D. M.; HUTZLER, B. W.; LANDGRAF, M. Combination of minimal processing and irradiation to improve the microbiological safety of lettuce (Lactuca sativa, L.). Radiation Physics and Chemistry, v. 71, p. 155-159, 2004.

11. GOULD, G. W. Methods for preservation and extension of shelf life. International Journal of Food Microbiology, v. 33, p. 51-64, 1996.

12. HERNANDES, N. K.; VITAL, H. C.; SABAA SRUR, A. U. O. Irradiação de alimentos: vantagens e limitações. Boletim SBCTA, v. 37, n. 2, p. 154-159, jul./dez. 2003.

13. KLUGE, R. A.; COSTA, C.; VITTI, M. C. D.; ONGARELLI, M. G.; JACOMINO, A. P.; MORETTI, C. L. Armazenamento refrigerado de beterraba minimamente processada em diferentes tipos de corte. Ciência Rural, v. 36, n. 1, p. 263-270, jan./ fev. 2006.

14. LANA, M. M. Aspectos da fisiologia de cenoura minimamente processada. Horticultura Brasileira, v. 18, n. 3, p. 154-158, novembro 2000.

15. LANGE, W.; BRANDENBURG, W. A.; DE BOCK, T. S. M. Taxonomy and cultonomy of beet (Beta vulgaris L.). Botanical Journal of the Linnean Society, v. 130, p. 81-96, 1999.

16. LIMA, K. S. C. Qualidade em cenouras (Daucus carota L.) cv. Nantes minimamente processadas e irradiadas: Avaliação química, física e microbiológica - Tese submetida como requisito parcial para obtenção do grau de Doutor em Ciência e Tecnologia de Alimentos - Universidade Federal Rural do Rio de Janeiro - Instituto de Tecnologia - Departamento de Tecnologia de Alimentos - Curso de Pós- Graduação em Ciência e Tecnologia de Alimentos. Seropédica, RJ, 2001.

17. LIMA, K. S. C; LIMA, A. L. S; FREITAS, L. C; DELLA-MODESTA, R. C; GODOY, R. L. O. - Efeito de baixas doses de irradiação nos carotenóides majoritários em cenouras prontas para o consumo. Ciência e Tecnologia de Alimentos, v. 24, n. 2, p. 183-193, abr./jun. 2004.

18. MAÑAS, P; PAGÁN, R. Microbial inactivation by new technologies of food preservation - A Review. Journal of Applied Microbiology, v. 98 , p. $1387-1399,2005$.
19. MARTINS, C. G.; BEHRENS, J. H.; DESTRO, M. T.; FRANCO, B. D. G. M.; VIZEU, D. M.; HUTZLER, B.; LANDGRAF, M. Gamma radiation in the reduction of Salmonella spp. inoculated on minimally processed watercress (Nasturtium officinalis). Radiation Physics and Chemistry, v. 71, p. 87-91, 2004.

20. MENEZES, E. M. S.; FERNANDES, E. C.; SABAA-SRUR, A. U. O. Folhas de alface lisa (Lactuca sativa) minimamente processadas armazenadas em atmosfera modificada: análises físicas, químicas e físico-químicas. Ciência e Tecnologia de Alimentos, v. 25, n. 1, p. 60-62, jan./mar. 2005.

21. OMS (Organización Mundial de la Salud). La irradiación de los alimentos - Una técnica para conservar y preservar la inocuidad de los alimentos, Genebra, 1989. 90 p. ISBN 92-4354-240-0.

22. OMS (Organización Mundial de la Salud). Inocuidad e idoneidad nutricional de los alimentos irradiados, Genebra, 1995. $172 \mathrm{p}$. ISBN 92-4-356162-6.

23. PASCH, J. H.; VON ELBE, J. H. Betanine stability in buffered solutions containing organic acids, metal cations, antioxidants, or sequestrants. Journal of food Science, v. 44, n. 1, p. 72-74,81, 1979.

24. RINAldi, M. M.; BEnEdetTI, B. C.; CALORE, L. Efeito da embalagem e temperatura de armazenamento em repolho minimamente processado. Ciência e Tecnologia de Alimentos, v. 25, n. 3, p. 480-486, jul./set. 2005.

25. SILVA, G. C.; MAIA, G. A.; FIGUEIREDO, R. W.; SOUZA FILHO, M. S. M.; ALVES, R. E.; SOUZA NETO, M. A. Efeito do tipo de corte nas características físico-químicas e físicas do abacaxi pérola minimamente processado. Ciência e Tecnologia de Alimentos, v. 25, n. 2, p. 223-228, abr.jun. 2005.

26. SOLIVA-FORTUNY, R. C.; MARTÍN-BELLOSO, O. New Advances in Extending the Shelf-life of Fresh-cut Fruits: a Review. Trends in Food Science \& Technology, v. 14, p. 341-353, 2003.

27. VITAL, H. C.; PIRES, L. F. G.; LIMA, R. Q.; VELLOZO, S. O. Experimentos dosimétricos no irradiador gama do IPE. Trabalho apresentado no $5^{\circ}$ Encontro Nacional de Aplicações Nucleares (ENAN), Rio de Janeiro, RJ, Brasil, 15 a 20 de Outubro de 2000.

28. VITTI, M. C. D. Aspectos fisiológicos, bioquímicos e microbiológicos em beterrabas minimamente processadas - Dissertação apresentada à Escola Superior de Agricultura "Luiz de Queiroz", Universidade de São Paulo, para obtenção do título de Mestre em Ciências, área de concentração: Fisiologia e Bioquímica de plantas - Piracicaba, SP, 2003.

29. VITTI, M. C. D.; KLUGE, R. A.; GALlO, C. R.; MORETTI, C. L.; JACOMINO, A. P. Efeito do momento de sanitização sobre atributos físico-químicos e microbiológicos de beterrabas minimamente processadas. Horticultura Brasileira, v. 22, n. 4, p. 718-721, out./dez. 2004. 